\title{
Construct validity of patient-reported outcome instruments in US adults with hemophilia: results from the Pain, Functional Impairment, and Quality of life (P-FiQ) study
}

\author{
This article was published in the following Dove Press journal: \\ Patient Preference and Adherence \\ 9 August 2017 \\ Number of times this article has been viewed
}

\section{Katharine Batt ${ }^{1}$ \\ Michael Recht ${ }^{2}$ \\ David L Cooper ${ }^{3}$ \\ Neeraj $\mathrm{N}$ lyer $^{3}$ \\ Christine L Kempton ${ }^{4}$}

'Hematology and Oncology, Wake Forest School of Medicine, WinstonSalem, NC, ${ }^{2}$ The Hemophilia Center, Oregon Health \& Science University, Portland, OR, ${ }^{3}$ Novo Nordisk Inc., Plainsboro, NJ, ${ }^{4}$ Departments of Pediatrics and Hematology and Medical Oncology, Emory University School of Medicine, Atlanta, GA, USA
Correspondence: Katharine Batt Wake Forest University Baptist Medical Center, Medical Center Boulevard, Winston-Salem, NC 27I0I, USA

Tel +l 3367160443

Fax +I 3367135445

Email kbatt@wakehealth.edu
Background: People with hemophilia (PWH) experience frequent joint bleeding, resulting in pain and functional impairment. Generic and disease-specific patient-reported outcome (PRO) instruments have been used in clinical studies, but rarely in the comprehensive hemophilia care setting. Objective: The objective of this study was to assess construct validity of PRO instruments measuring pain, functional impairment, and health-related quality of life in US PWH with a history of joint pain/bleeding.

Methods: Adult male PWH completed 4 PRO instruments (EQ-5D-5L with visual analog scale, Brief Pain Inventory v2 Short Form [BPI], SF-36v2, Hemophilia Activities List [HAL]) and underwent a musculoskeletal examination (Hemophilia Joint Health Score v2.1 [HJHS]). Construct validity between index and domain scores was evaluated by Pearson product-moment correlation coefficient.

Results: A total of 381 PWH were enrolled. EQ-5D-5L Mobility correlated with BPI, SF-36v2, and HAL domains related to pain, physical function, and activity of the lower extremities. EQ-5D-5L Self-Care correlated only with HAL Self-Care. EQ-5D-5L Usual Activities correlated with BPI Pain Interference and domains within SF-36v2 and HAL related to pain and physical function/ activities (particularly those involving the lower extremities). EQ-5D-5L Pain/Discomfort correlated with Bodily Pain and Physical Summary on SF-36v2, HAL Overall Activity, and all BPI pain domains. EQ-5D-5L Anxiety/Depression correlated with social/emotional/mental aspects of SF-36v2. On BPI, most pain domains correlated with Bodily Pain and Physical Health Summary on SF-36v2 and Overall Activity on HAL. On SF-36v2, Physical Functioning, Role Physical, Bodily Pain, and Physical Health summary scores correlated with all the domains of HAL except Self-Care. For HJHS, Ankle and Total scores correlated with SF-36v2 Physical Functioning and HAL Lying/Sitting, Leg Function, Complex Lower Extremity Activity, and Overall Activity. Conclusion: All PRO instruments have high construct validity but provide different levels of detail in describing effects of hemophilia. Instrument choice may depend on individuals' symptoms, treatment planning goals, or outcome tracking research objectives, with consideration for administrative burden.

Keywords: hemophilia, patient-reported outcomes, construct validity, pain, functional impairment, quality of life

\section{Introduction}

Congenital hemophilia is a rare bleeding disorder caused by a deficiency in either factor VIII (hemophilia A) or factor IX (hemophilia B), preventing proper activation of the 
clotting cascade and resulting in prolonged or excessive bleeding. ${ }^{1-3}$ A characteristic feature of hemophilia is frequent bleeding into joints (hemarthrosis), which leads to progressive joint damage and may cause significant pain, functional impairment, and diminished health-related quality of life (HRQoL). ${ }^{4-6}$ As the treatment of hemophilia has advanced rapidly over the last several decades and the average life expectancy for people with hemophilia (PWH) has risen, improved assessment and management of disease comorbidities has become an important focus in improving comprehensive care of adult PWH.

Despite the importance of accurately measuring pain and its effects on HRQoL in PWH, assessment of patient-reported outcomes (PROs) has been limited and inconsistent in the clinical care setting. ${ }^{7-9}$ Although some generic PRO instruments such as EQ-5D and SF-36 have been used in PWH, this has occurred primarily within the context of clinical studies, and these instruments have not been specifically validated for use in the hemophilia patient population. ${ }^{6,10}$ The Brief Pain Inventory v2 Short Form (BPI) is an attractive option for the specific and detailed assessment of pain, as it measures both pain severity and pain interference and has been validated in both cancer and non-cancer pain; ${ }^{11-14}$ however, use of this instrument in adult PWH has not yet been reported outside of the Pain, Functional Impairment, and Quality of Life (P-FiQ) study. An important aspect of HRQoL for assessment in $\mathrm{PWH}$ is functional impairment resulting from pain and joint disease. Because functional impairment in adult PWH typically manifests across specific types of activities, a hemophilia-specific functional assessment tool (the Hemophilia Activities List [HAL]) was previously designed with input from focus groups of patients in the Netherlands who identified activities with which they experienced difficulties. The HAL may be a useful tool within the clinical and research settings, because it measures specific functional limitations relevant to hemophilic arthropathy, but it has not yet been validated in an English-speaking population, and it is infrequently used for individual outcome assessment. ${ }^{15,16}$ Given the current lack of consensus regarding the measurement of pain and HRQoL in PWH, validating PRO scales is an important step toward facilitating the incorporation of these assessments into comprehensive clinical care.

The P-FiQ study was a US-based, non-interventional, cross-sectional assessment of the impact of pain on functional impairment and HRQoL in adult PWH with a history of joint pain or bleeding. The study included the administration of 5 generic and disease-specific PRO instruments (EQ-5D-5L,
BPI, International Physical Activity Questionnaire [IPAQ], SF-36v2, and HAL) with the potential for adoption into clinical practice and research, as well as a physical therapistadministered musculoskeletal examination (Hemophilia Joint Health Score [HJHS]). Each of these instruments differs in scope, level of detail, timescale of assessment, and administrative burden, which may influence the applicability for use in specific care settings and patient circumstances. Although previous reports have presented the overall PRO data ${ }^{17}$ and analysis of test-retest reliability, ${ }^{18}$ this study reports data evaluating construct validity, which may contribute to a greater understanding of whether these instruments accurately measure constructs related to pain, functional impairment, and other aspects of HRQoL.

\section{Methods}

\section{Recruitment and study design}

Adult male PWH who were attending an annual or other routine comprehensive care visit were approached for enrollment in P-FiQ. The study protocol was approved by the local institutional review board (IRB) or central IRB. A list of all 14 approving IRBs is provided in the Supplementary materials. Investigators obtained written informed consent from the participants prior to engaging in any study-related activities. Participants could withdraw the consent at any time without obligation or penalty, and all were compensated for their participation in the study.

Eligibility criteria required all participants to be male, aged $\geq 18$ years with congenital hemophilia A or B (with or without inhibitors) and a history of joint pain or bleeding, attending a scheduled visit to a hemophilia treatment center (eg, an annual comprehensive care or other routine visit) during which joint range of motion would be assessed, able to provide informed consent, and able to complete the study survey in English. Individuals who had previously participated in P-FiQ were not eligible for subsequent reenrollment.

Participation in the study included completion of a survey on sociodemographics, a pain history, and 5 PRO instruments (EQ-5D-5L with visual analog scale [VAS], BPI, IPAQ, SF-36v2, and HAL), which were administered prior to the scheduled care visit. During the scheduled care visit, the site personnel completed a questionnaire regarding the participants' medical history, and sites with available trained physical therapists optionally completed a standardized musculoskeletal examination (HJHS). Construct validity was assessed for 4 PRO instruments (EQ-5D-5L, BPI, SF-36v2, and HAL) and HJHS. 


\section{Patient outcome assessment tools EQ-5D-5L}

The EQ-5D-5L is a PRO instrument that was developed in 2011 for use as a simple, generic measure of health outcomes for a wide range of health conditions and treatments. ${ }^{19}$ This instrument consists of 2 sections, the EQ-5D-5L descriptive system and the 100-point VAS, each of which measures health status "today." The descriptive section consists of 5 dimensions (Mobility, Self-Care, Usual Activities, Pain/ Discomfort, and Anxiety/Depression), each described by 5 response levels (no, slight, moderate, severe, or extreme problems), as well as an index score that is calculated from individual descriptive responses.

\section{BPI}

The BPI is a generic PRO instrument that was developed in 1994 to assess the severity of pain and the impact of pain on daily functions among adults. ${ }^{12}$ For the P-FiQ study, the BPI was used to assess the pain experienced over the past week. This assessment tool consists of 2 sections: Pain Severity, calculated as the average of 4 individual domains (Worst Pain, Least Pain, Average Pain, and Current Pain), and Pain Interference, calculated as the average of 7 individual domains (General Activity, Mood, Walking Ability, Normal Work, Relations with Other People, Sleep, and Enjoyment of Life). Each domain is measured on a scale from 0 ("no pain" or "does not interfere") to 10 ("pain as bad as you can imagine" or "completely interferes").

\section{SF-36v2}

The SF-36v2 is a generic PRO instrument that was developed in 2010 to measure health concepts that are relevant across different ages, diseases, and treatment groups. ${ }^{20}$ The instrument consists of a Physical Health Summary, composed of 4 domains (Physical Function, Role Physical, Bodily Pain, and General Health), a Mental Health Summary, composed of 4 domains (Vitality, Social Functioning, Role Emotional, and Mental Health), and an Overall Health summary score. Each domain measures health status as experienced over the past 4 weeks using a 100-point scale.

\section{HAL}

The HAL is a disease-specific PRO instrument that was developed in 2004 based on initial surveys of adults with hemophilia ${ }^{15}$ and has been validated in the Netherlands, ${ }^{16}$ but not in PWH from native English-speaking countries. The instrument consists of 7 domains measuring various aspects of physical function as experienced over the past month (Lying/Sitting/Kneeling/Standing, Functions of the Legs, Functions of the Arms, Use of Transportation, SelfCare, Household Tasks, and Leisure Activities and Sports) using a 0 - to 100-point scale. Summary scores (Upper Extremity Activities, Basic Lower Extremity Activities, Complex Lower Extremity Activities, and Overall Sum) are also derived from relevant domain scores.

\section{HJHS}

The HJHS is a physical therapist-administered instrument that was developed in 2011 to measure joint health status and to identify early signs of joint disease in children. This tool assesses joints most commonly affected by bleeding in PWH (knees, ankles, and elbows). ${ }^{21,22}$ Each of the 6 joints is assessed across 8 domains (Swelling, Duration of Swelling, Atrophy, Crepitus, Flexion Loss, Extension Loss, Pain, and Strength), and a Total Score is calculated as the sum of all joint domain scores. The instrument also includes a measure of Global Gait, which encompasses assessments of 4 skills: walking, stairs, running, and hopping on 1 leg.

\section{Data analysis}

Construct validity was measured by Pearson productmoment correlation coefficient $(r)$, with corresponding $P$-values. Correlation coefficients can be categorized as small $(r=0.1-0.23)$, moderate $(r=0.24-0.36)$, and high $(r \geq 0.37),{ }^{23}$ this analysis and report are focused on the strongest correlations (PRO instruments, $r>0.6$; HJHS, $r>0.5$ ). All statistical analyses were performed using SAS software, version 9.2 or higher (SAS Institute, Cary, NC, USA).

\section{Results \\ Participant characteristics}

Overall, 381 adult male PWH were enrolled between October 2013 and October 2014 from 15 separate trial sites. Mean (median) enrollment per site was 25.4 (28) subjects, and none withdrew from the study. Median age was 34 years, and most (77.4\%) of the participants had hemophilia A; 22.6\% had hemophilia B, and $8.7 \%$ had inhibitors. A majority of participants had severe hemophilia (factor VIII or factor IX level $<1 \%$; 70.5\%), and most reported experiencing pain during the 6 months preceding study enrollment (any pain, $85.3 \%$; acute pain only, $19.5 \%$; chronic pain only, $34.4 \%$; acute and chronic pain, $31.5 \%$ ). Ankles were most often reported as the most painful joints (37\%), followed by knees (24\%) and elbows (19\%). Arthritis/bone/joint problems were 
self-reported by $64.6 \% ; 64.8 \%$ were overweight or obese. Functional disability was assessed using the Centers for Disease Control and Prevention Universal Data Collection template, ${ }^{24}$ on which many participants reported limitations in school/ work or recreational activities due to pain, loss of motion, or weakness (66.6\%). Anxiety and depression were self-reported by $13.9 \%$ and $16.3 \%$ of the participants, respectively.

\section{EQ-5D-5L}

Correlations between EQ-5D-5L domains (including VAS and index score) and other PRO domain and summary scores are presented in Table 1. Of 196 individual comparisons, strong correlations $(P<0.001)$ were observed for all except for EQ-5D-5L Anxiety/Depression with SF-36v2 Overall Health $(P=0.012)$. All correlations reflect a directionality of lower scores (indicating worsening HRQoL) correlating with other measures of worse outcomes.

The highest correlations ( $r>0.6)$ observed for EQ-5D-5L Mobility were with domain/summary scores expected to affect mobility, including various BPI, SF-36v2, and HAL scores related to pain, physical function, and activities of the lower extremities. EQ-5D-5L Usual Activities showed

Table I Correlation of EQ-5D-5L domains with BPI, SF-36v2, and HAL scores

\begin{tabular}{|c|c|c|c|c|c|c|c|}
\hline \multirow[t]{2}{*}{ Construct } & \multicolumn{7}{|c|}{ Pearson's $r$} \\
\hline & Mobility & $\begin{array}{l}\text { Self- } \\
\text { Care }\end{array}$ & $\begin{array}{l}\text { Usual } \\
\text { Activities }\end{array}$ & $\begin{array}{l}\text { Pain/ } \\
\text { Discomfort }\end{array}$ & $\begin{array}{l}\text { Anxietyl } \\
\text { Depression }\end{array}$ & Index & $\begin{array}{l}\text { Visual analog } \\
\text { scale }\end{array}$ \\
\hline \multicolumn{8}{|l|}{ BPI } \\
\hline \multicolumn{8}{|l|}{ Domain scores } \\
\hline Worst pain & 0.56 & 0.34 & 0.50 & 0.69 & 0.39 & -0.66 & -0.55 \\
\hline Least pain & 0.53 & 0.31 & 0.50 & 0.64 & 0.35 & -0.59 & -0.49 \\
\hline Average pain & 0.61 & 0.34 & 0.51 & 0.72 & $0.4 I$ & -0.67 & -0.54 \\
\hline Current pain & 0.65 & 0.41 & 0.56 & 0.77 & 0.45 & -0.74 & -0.62 \\
\hline \multicolumn{8}{|l|}{ Summary scores } \\
\hline Pain severity & 0.65 & 0.39 & 0.57 & 0.78 & 0.44 & -0.74 & $-0.6 \mathrm{I}$ \\
\hline Pain interference & 0.62 & 0.43 & 0.63 & 0.68 & 0.45 & -0.70 & -0.62 \\
\hline \multicolumn{8}{|l|}{ SF-36v2 } \\
\hline \multicolumn{8}{|l|}{ Domain scores } \\
\hline Physical functioning & -0.73 & -0.50 & -0.63 & -0.58 & -0.29 & 0.65 & 0.57 \\
\hline Role physical & -0.62 & -0.47 & -0.66 & -0.57 & -0.39 & 0.64 & 0.59 \\
\hline Bodily pain & -0.64 & -0.45 & -0.64 & -0.72 & -0.40 & 0.73 & 0.60 \\
\hline General health & -0.51 & -0.35 & -0.47 & -0.53 & -0.43 & 0.59 & 0.69 \\
\hline Vitality & -0.41 & -0.30 & -0.45 & -0.48 & -0.50 & 0.53 & 0.55 \\
\hline Social functioning & -0.53 & -0.43 & -0.57 & -0.55 & -0.61 & 0.68 & 0.59 \\
\hline Role emotional & -0.38 & -0.30 & -0.42 & -0.41 & -0.63 & 0.55 & 0.43 \\
\hline Mental health & -0.26 & -0.23 & -0.35 & -0.37 & $-0.7 \mathrm{I}$ & 0.47 & 0.42 \\
\hline \multicolumn{8}{|l|}{ Summary scores } \\
\hline Physical health summary & -0.72 & -0.49 & -0.66 & -0.64 & -0.22 & 0.66 & 0.64 \\
\hline Mental health summary & -0.21 & -0.19 & -0.30 & -0.33 & -0.72 & 0.45 & 0.39 \\
\hline Overall health & 0.29 & 0.22 & 0.31 & 0.29 & 0.13 & -0.29 & -0.42 \\
\hline \multicolumn{8}{|l|}{ HAL } \\
\hline \multicolumn{8}{|l|}{ Domain scores } \\
\hline Lying/sitting/kneeling/standing & -0.66 & -0.40 & -0.57 & -0.59 & -0.33 & 0.62 & 0.49 \\
\hline Functions of the legs & -0.73 & -0.36 & -0.60 & -0.58 & -0.32 & 0.64 & 0.52 \\
\hline Functions of the arms & -0.52 & -0.45 & $-0.5 \mathrm{I}$ & -0.52 & -0.35 & 0.55 & 0.46 \\
\hline Use of transportation & -0.63 & -0.52 & -0.58 & -0.55 & -0.31 & 0.60 & 0.53 \\
\hline Self-care & -0.46 & -0.62 & $-0.5 \mathrm{I}$ & -0.45 & -0.32 & 0.52 & 0.39 \\
\hline Household tasks & -0.62 & $-0.5 \mathrm{I}$ & -0.62 & -0.58 & -0.39 & 0.64 & 0.55 \\
\hline Leisure activities and sports & -0.64 & -0.43 & -0.64 & -0.52 & -0.34 & 0.60 & 0.48 \\
\hline \multicolumn{8}{|l|}{ Summary scores } \\
\hline Upper extremity activities & -0.53 & -0.57 & -0.56 & -0.53 & -0.37 & 0.59 & 0.47 \\
\hline Basic lower extremity activities & -0.75 & -0.35 & -0.61 & -0.59 & -0.34 & 0.65 & 0.53 \\
\hline Complex lower extremity activities & -0.68 & -0.37 & -0.56 & -0.58 & -0.29 & 0.62 & 0.49 \\
\hline Overall activity & -0.72 & -0.49 & -0.66 & -0.63 & -0.38 & 0.69 & 0.56 \\
\hline
\end{tabular}

Notes: High correlations are defined as $r \geq 0.37$. Bold values indicate the strongest correlations with $r>0.6$. $P \leq 0.001$ for all comparisons except EQ-5D-5L Anxiety/ Depression versus SF-36v2 Overall Health $(P=0.012)$.

Abbreviations: BPI, Brief Pain Inventory v2 Short Form; HAL, Hemophilia Activities List. 
very strong correlations with BPI Pain Interference, SF-36v2 scores related to physical pain and function, and HAL scores related to functions of the legs (Functions of the Legs and Basic Lower Extremity Activities) and usual activities (Household Tasks, Leisure Activities and Sports, and Overall Activity). EQ-5D-5L Pain/Discomfort correlated most strongly with pain-specific scores, including all BPI domain/ summary scores, and with HAL Overall Activity. EQ-5D-5L Self-Care demonstrated a very strong correlation only with HAL Self-Care, and EQ-5D-5L Anxiety/Depression correlated with SF-36v2 Social Functioning, Role Emotional, and Mental Health scores. EQ-5D-5L VAS scores (measuring health "today") correlated most strongly with other measures of health status (SF-36v2 General Health and Physical Health Summary) and with specific measures of pain (including Current Pain on BPI, as well as both Pain Severity and Pain Interference). EQ-5D-5L Index scores showed very strong correlations with several domain/summary scores, including most BPI pain scores, SF-36v2 scores related to physical and social function, and HAL scores related to functions of the lower extremities.

Select EQ-5D-5L correlations between scores that were expected to be related are graphically depicted in Figure 1. These data demonstrate a significant correlation between worsening EQ-5D-5L Mobility assessments and worsening HAL Functions of the Legs scores (Figure 1A). Similarly, separate measures of self-care (EQ-5D-5L Self-Care and HAL Self-Care) were shown to correlate significantly (Figure 1B), suggesting high convergent validity for these assessment tools. Select correlations of EQ-5D-5L Pain/ Discomfort are additionally presented in Supplementary materials, which demonstrate significant correlations with various measures of pain and physical function (BPI Pain Severity and Pain Interference [Figure S1], SF-36v2 Bodily Pain [Figure S2], and HAL Upper Extremity Activities, Basic Lower Extremity Activities, and Complex Lower Extremity Activities [Figure S3]).

\section{BPI}

Correlations between each of the 4 BPI pain domains (Worst Pain, Least Pain, Average Pain, and Current Pain) with SF-36v2 and HAL domain/summary scores are presented in Table 2. All correlations reached significance of $P<0.001$, and all reflected a directionality of higher scores (indicating worsening pain) correlating with other measures of worse outcomes. Each BPI domain had a strong correlation $(r>0.6)$ with SF-36v2 Bodily Pain; most BPI domains (all except for Least Pain) also correlated highly with SF-36v2 Physical
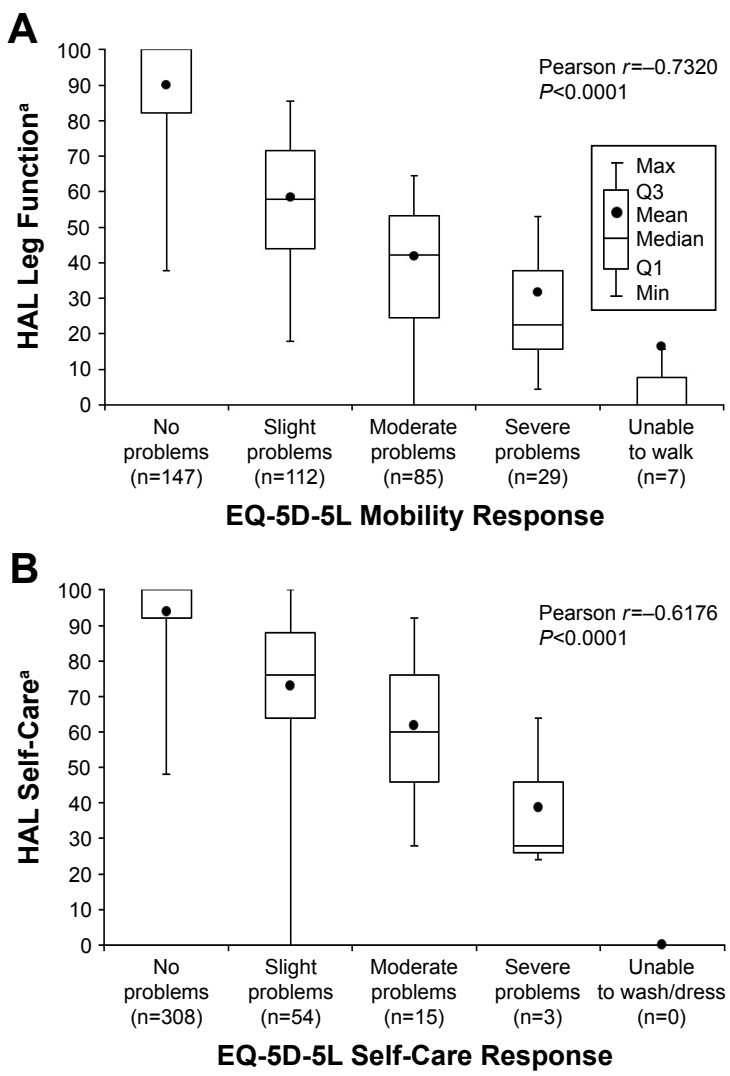

Figure I Correlation of EQ-5D-5L Mobility with HAL Leg Function (A) and of EQ5D-5L Self-Care with HAL Self-Care (B).

Notes: ${ }^{a} \mathrm{HAL}$ scores measured on a scale from 0 to 100 ; higher scores indicate better functional status.

Abbreviation: HAL, Hemophilia Activities List.

Health Summary and with HAL Overall Activity. In addition, current pain, but not the other BPI domains, was shown to correlate highly with HAL Household Tasks.

\section{SF-36v2}

Correlations between the 8 domain scores and 3 summary scores of SF-36v2 with HAL domain/summary scores are presented in Table 3. All correlations reached significance of $P<0.001$, and the directionality of each demonstrated higher scores (indicating worse HRQoL) correlating with other measures of poorer outcomes.

SF-36v2 Physical Functioning, Role Physical, and Bodily Pain each correlated highly $(r>0.6)$ with all measures of physical function on HAL, with the exceptions of HAL SelfCare, Functions of the Arms (did not reach $r>0.6$ with Role Physical and Bodily Pain) and Upper Extremity Activities (did not reach $r>0.6$ with Bodily Pain). In contrast, SF-36v2 Social Functioning correlated strongly with HAL scores related to activities of the upper extremities (Functions of the Arms, Household Tasks, Leisure Activities and Sports, Upper Extremity Activities, and Overall Activity), and SF-36v2 
Table 2 Correlation of BPI domains with SF-36v2 and HAL scores

\begin{tabular}{|c|c|c|c|c|}
\hline \multirow[t]{2}{*}{ Construct } & \multicolumn{4}{|c|}{ Pearson's $r$} \\
\hline & $\begin{array}{l}\text { Worst } \\
\text { Pain }\end{array}$ & $\begin{array}{l}\text { Least } \\
\text { Pain }\end{array}$ & $\begin{array}{l}\text { Average } \\
\text { Pain }\end{array}$ & $\begin{array}{l}\text { Current } \\
\text { Pain }\end{array}$ \\
\hline \multicolumn{5}{|l|}{ SF-36v2 } \\
\hline \multicolumn{5}{|l|}{ Domain scores } \\
\hline Physical functioning & -0.52 & -0.54 & -0.56 & -0.58 \\
\hline Role physical & -0.53 & -0.49 & -0.53 & -0.55 \\
\hline Bodily pain & -0.77 & -0.63 & -0.75 & -0.72 \\
\hline General health & -0.52 & -0.52 & -0.54 & -0.57 \\
\hline Vitality & -0.50 & -0.39 & -0.47 & -0.48 \\
\hline Social functioning & -0.54 & -0.52 & -0.59 & -0.58 \\
\hline Role emotional & -0.38 & -0.45 & -0.44 & -0.44 \\
\hline Mental health & -0.39 & -0.32 & -0.40 & -0.41 \\
\hline \multicolumn{5}{|l|}{ Summary scores } \\
\hline Physical health summary & -0.61 & -0.56 & -0.61 & -0.62 \\
\hline Mental health summary & -0.33 & -0.33 & -0.37 & -0.37 \\
\hline Overall health & 0.34 & 0.25 & 0.31 & 0.34 \\
\hline \multicolumn{5}{|l|}{ HAL } \\
\hline \multicolumn{5}{|l|}{ Domain scores } \\
\hline Lying/sitting/kneeling/standing & -0.57 & -0.53 & -0.57 & -0.60 \\
\hline Functions of the legs & -0.58 & -0.49 & -0.55 & -0.58 \\
\hline Functions of the arms & -0.48 & -0.51 & -0.51 & -0.54 \\
\hline Use of transportation & -0.49 & -0.48 & -0.51 & -0.56 \\
\hline Self-care & -0.42 & $-0.4 \mathrm{I}$ & -0.44 & -0.48 \\
\hline Household tasks & -0.55 & -0.53 & -0.55 & -0.61 \\
\hline Leisure activities and sports & -0.53 & -0.48 & -0.51 & -0.55 \\
\hline \multicolumn{5}{|l|}{ Summary scores } \\
\hline Upper extremity activities & -0.49 & -0.50 & -0.52 & -0.55 \\
\hline $\begin{array}{l}\text { Basic lower extremity } \\
\text { activities }\end{array}$ & -0.58 & -0.52 & -0.57 & -0.59 \\
\hline $\begin{array}{l}\text { Complex lower extremity } \\
\text { activities }\end{array}$ & -0.57 & -0.48 & -0.54 & -0.56 \\
\hline Overall activity & -0.61 & -0.56 & -0.60 & -0.64 \\
\hline
\end{tabular}

Notes: High correlations are defined as $r \geq 0.37$. Bold values indicate the strongest correlations with $r>0.6$. $P<0.00$ I for all comparisons.

Abbreviations: BPI, Brief Pain Inventory v2 Short Form; HAL, Hemophilia Activities List.

General Health showed a strong correlation only with HAL Overall Activity. SF-36v2 Vitality, Role Emotional, and Mental Health did not exhibit strong correlations with any HAL domain/summary scores. Of the 3 SF-36v2 summary scores, Mental Health Summary and Overall Health did not reach strong correlations with any HAL domain/summary scores; however, Physical Health Summary correlated very strongly with all HAL scores except for Self-Care.

\section{HJHS}

An optional HJHS examination was performed for 240 participants (63\%). Correlations between HJHS domain/ summary scores and PRO instrument scores are presented in Table 4. All correlations reached a significance of $P<0.01$, except for those of each domain/summary score with EQ-5D-5L Anxiety/Depression and with SF-36v2 Overall Health. All correlations reflect a directionality of higher scores

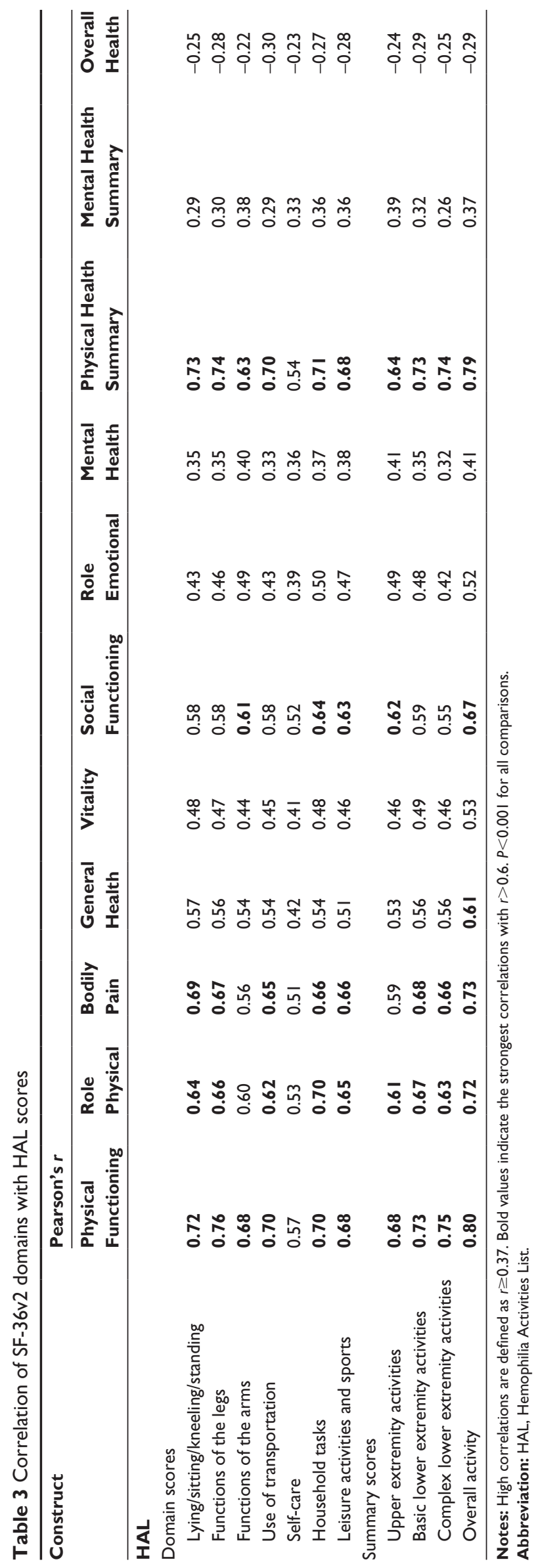


Table 4 Correlation of HJHS domains with EQ-5D-5L, BPI, SF-36v2, and HAL scores

\begin{tabular}{|c|c|c|c|c|c|c|c|c|c|c|}
\hline \multirow[t]{2}{*}{ Construct } & \multicolumn{2}{|l|}{ Elbow } & \multicolumn{2}{|l|}{ Ankle } & \multicolumn{2}{|l|}{ Knee } & \multicolumn{2}{|l|}{ Global Gait } & \multicolumn{2}{|l|}{ Total Score } \\
\hline & Pearson's $r$ & $P$-value & Pearson's $r$ & $P$-value & Pearson's $r$ & $P$-value & Pearson's r & $P$-value & Pearson's $r$ & $P$-value \\
\hline \multicolumn{11}{|l|}{ EQ-5D-5L } \\
\hline \multicolumn{11}{|l|}{ Domain scores } \\
\hline Mobility & 0.39 & $<0.01$ & 0.50 & $<0.01$ & 0.40 & $<0.01$ & 0.22 & $<0.01$ & 0.50 & $<0.01$ \\
\hline Self-care & 0.36 & $<0.01$ & 0.33 & $<0.01$ & 0.19 & $<0.01$ & 0.12 & $<0.01$ & 0.34 & $<0.01$ \\
\hline Usual activities & 0.33 & $<0.01$ & 0.39 & $<0.01$ & 0.30 & $<0.01$ & 0.06 & $<0.01$ & 0.40 & $<0.01$ \\
\hline Pain/discomfort & 0.37 & $<0.01$ & 0.39 & $<0.01$ & 0.29 & $<0.01$ & 0.03 & $<0.01$ & 0.41 & $<0.0$ I \\
\hline Anxiety/depression & 0.16 & 0.01 & 0.13 & 0.04 & 0.08 & 0.24 & 0.11 & 0.24 & 0.14 & 0.03 \\
\hline \multicolumn{11}{|l|}{ Summary scores } \\
\hline Index & -0.40 & $<0.01$ & -0.45 & $<0.01$ & -0.30 & $<0.01$ & -0.17 & $<0.01$ & -0.45 & $<0.01$ \\
\hline Visual analog scale & -0.35 & $<0.01$ & -0.28 & $<0.01$ & -0.24 & $<0.01$ & -0.13 & $<0.01$ & -0.33 & $<0.001$ \\
\hline \multicolumn{11}{|l|}{ BPI } \\
\hline \multicolumn{11}{|l|}{ Domain scores } \\
\hline Worst pain & 0.37 & $<0.01$ & 0.37 & $<0.01$ & 0.40 & $<0.01$ & 0.20 & $<0.01$ & 0.44 & $<0.01$ \\
\hline Least pain & 0.25 & $<0.01$ & 0.37 & $<0.01$ & 0.29 & $<0.0 \mathrm{I}$ & -0.02 & $<0.01$ & 0.35 & $<0.0 \mathrm{I}$ \\
\hline Average pain & 0.35 & $<0.01$ & 0.39 & $<0.01$ & 0.38 & $<0.01$ & 0.10 & $<0.01$ & 0.44 & $<0.01$ \\
\hline Current pain & 0.35 & $<0.01$ & 0.42 & $<0.01$ & 0.28 & $<0.01$ & 0.14 & $<0.01$ & 0.40 & $<0.01$ \\
\hline \multicolumn{11}{|l|}{ Summary scores } \\
\hline Pain severity & 0.37 & $<0.01$ & 0.43 & $<0.01$ & 0.38 & $<0.01$ & 0.13 & $<0.01$ & 0.45 & $<0.0 \mathrm{I}$ \\
\hline Pain interference & 0.35 & $<0.01$ & 0.33 & $<0.01$ & 0.30 & $<0.0 \mathrm{I}$ & 0.17 & $<0.01$ & 0.38 & $<0.01$ \\
\hline \multicolumn{11}{|l|}{ SF-36v2 } \\
\hline \multicolumn{11}{|l|}{ Domain scores } \\
\hline Physical functioning & -0.41 & $<0.01$ & -0.53 & $<0.01$ & -0.38 & $<0.01$ & -0.18 & $<0.01$ & $-0.5 \mathrm{I}$ & $<0.0 \mathrm{I}$ \\
\hline Role physical & -0.37 & $<0.01$ & -0.36 & $<0.01$ & -0.28 & $<0.0 \mathrm{I}$ & -0.11 & $<0.01$ & -0.39 & $<0.01$ \\
\hline Bodily pain & -0.39 & $<0.01$ & -0.37 & $<0.01$ & -0.38 & $<0.01$ & -0.15 & $<0.01$ & -0.44 & $<0.01$ \\
\hline \multicolumn{11}{|l|}{ Summary scores } \\
\hline Physical health summary & -0.42 & $<0.01$ & -0.47 & $<0.01$ & -0.34 & $<0.01$ & -0.18 & $<0.01$ & -0.47 & $<0.01$ \\
\hline Overall health & 0.16 & 0.02 & 0.06 & 0.27 & 0.13 & 0.26 & 0.10 & 0.26 & 0.15 & 0.23 \\
\hline \multicolumn{11}{|l|}{ HAL } \\
\hline \multicolumn{11}{|l|}{ Domain scores } \\
\hline Lying/sitting/kneeling/standing & -0.44 & $<0.01$ & -0.56 & $<0.01$ & -0.40 & $<0.01$ & -0.05 & $<0.01$ & -0.53 & $<0.01$ \\
\hline Functions of the legs & -0.42 & $<0.01$ & $-0.5 I$ & $<0.01$ & -0.45 & $<0.01$ & -0.13 & $<0.01$ & -0.53 & $<0.01$ \\
\hline Functions of the arms & -0.44 & $<0.01$ & $-0.4 I$ & $<0.01$ & -0.27 & $<0.0 \mathrm{I}$ & -0.15 & $<0.01$ & -0.42 & $<0.01$ \\
\hline Use of transportation & -0.44 & $<0.01$ & -0.53 & $<0.01$ & -0.30 & $<0.01$ & -0.09 & $<0.01$ & -0.48 & $<0.01$ \\
\hline Self-care & -0.37 & $<0.01$ & -0.30 & $<0.01$ & -0.19 & $<0.01$ & -0.12 & $<0.01$ & -0.32 & $<0.01$ \\
\hline Household tasks & -0.43 & $<0.01$ & -0.42 & $<0.01$ & -0.28 & $<0.01$ & -0.12 & $<0.01$ & -0.42 & $<0.01$ \\
\hline Leisure activities and sports & -0.36 & $<0.01$ & -0.41 & $<0.01$ & -0.32 & $<0.01$ & -0.13 & $<0.01$ & $-0.4 I$ & $<0.01$ \\
\hline \multicolumn{11}{|l|}{ Summary scores } \\
\hline Upper extremity activities & -0.45 & $<0.01$ & -0.39 & $<0.01$ & -0.26 & $<0.01$ & -0.15 & $<0.01$ & -0.41 & $<0.01$ \\
\hline $\begin{array}{l}\text { Basic lower extremity } \\
\text { activities }\end{array}$ & -0.42 & $<0.01$ & -0.47 & $<0.01$ & -0.40 & $<0.01$ & -0.14 & $<0.01$ & -0.49 & $<0.01$ \\
\hline $\begin{array}{l}\text { Complex lower extremity } \\
\text { activities }\end{array}$ & -0.46 & $<0.01$ & -0.59 & $<0.01$ & -0.45 & $<0.01$ & -0.07 & $<0.01$ & -0.58 & $<0.01$ \\
\hline Overall activity & -0.47 & $<0.01$ & -0.53 & $<0.01$ & -0.39 & $<0.01$ & -0.12 & $<0.01$ & -0.53 & $<0.01$ \\
\hline
\end{tabular}

Notes: High correlations are defined as $r \geq 0.37$. Bold values indicate the strongest correlations with $r>0.5$.

Abbreviations: BPI, Brief Pain Inventory v2 Short Form; HAL, Hemophilia Activities List; HJHS, Hemophilia Joint Health Score v2.I.

(indicating worsening joint function) correlating with other measures of worse outcomes.

While correlations met the standard definition of high $(r \geq 0.37)$, no correlations reached the threshold of $r>0.6$ used in this analysis for consideration of reporting only strongest correlations; however, the HJHS Ankle domain reached a correlation of $r>0.5$ for several domain/summary scores related to physical function, including scores specific to activity of the lower extremities (SF-36v2 Physical Functioning and HAL Lying/Sitting/Kneeling/Standing, Functions of the Legs, Use of Transportation, Complex Lower Extremity Activities, and Overall Activity). HJHS Total Score also correlated at $r>0.5$ with most of these same PRO scores that correlated with HJHS Ankle (SF-36v2 Physical Functioning and HAL Lying/Sitting/Kneeling/Standing, Functions of the Legs, Complex Lower Extremity Activities, and Overall Activity). HJHS Global Gait did not reach a correlation of $r>0.5$ with any PRO instrument domain or summary scores. 


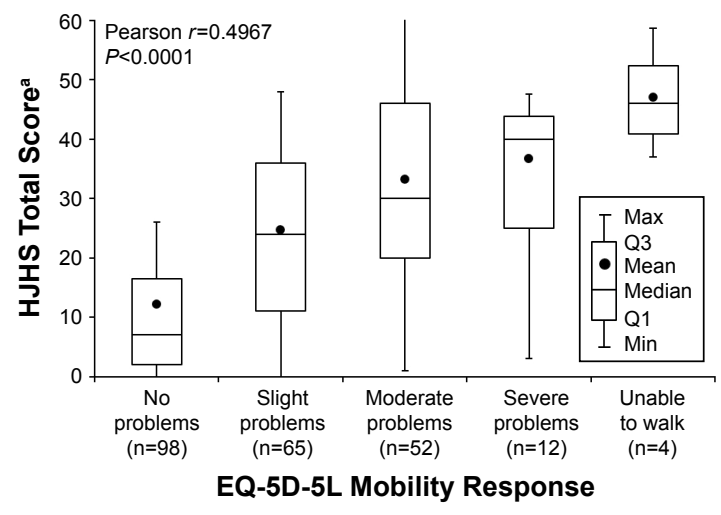

Figure 2 Correlation of EQ-5D-5L Mobility with HJHS Total Score. Notes: aHJHS Total Score measured on a scale from 0 to 124; lower scores indicate better joint function.

Abbreviation: HJHS, Hemophilia Joint Health Score v2.I.

The correlation between HJHS Total Score and EQ-5D-5L Mobility is graphically depicted in Figure 2, which demonstrates a significant correlation between increasing functional impairment and decreased joint health.

\section{Discussion}

Overall, this analysis demonstrated high construct validity of 5 PRO and physician-administered instruments that could be used for the assessment of pain, functional impairment, and HRQoL in adult PWH. Because reported experience with PRO instruments in this population has been limited and inconsistent to date, information regarding construct validity is important when making clinical decisions about whether to incorporate PRO instruments into comprehensive care.

As expected, high convergent validity of PROs was observed with directionality of the correlation as would be expected based on the direction of the PRO instruments (worse outcomes indicated by higher or lower scores); highly similar related domains (eg, separate measures of pain or specific physical limitations) exhibited the strongest correlations. These correlations were observed despite differences in the measured time frames between the different PRO instruments, which ranged from assessments of "today" on EQ-5D-5L to "in the previous month" on SF-36v2 and HAL. Independent measures of pain (EQ-5D-5L Pain/Discomfort and all BPI pain severity scores) were all highly correlated; furthermore, validity of the temporal specificity of these assessments is supported by a very strong correlation $(r>0.6)$ of EQ-5D VAS (measuring health "today") with BPI Current Pain, but not with Worst Pain, Least Pain, or Average Pain experienced during the past week. Independent measures of overall physical functioning (EQ-5D-5L Mobility and Usual Activities, SF-36v2 Physical Functioning, Role Physical, and Physical Health Summary, and HAL Overall Activity, Household Tasks, and Leisure Activities and Sports) were also all very strongly correlated, suggesting that each of these scores reflects an accurate measure of general physical function. Interestingly, while measures of self-care (EQ-5D-5L Self-Care and HAL Self-Care) showed relatively little impairment compared with other domains, ${ }^{17}$ they were correlated strongly only with each other. This suggests that self-care abilities may be relatively well preserved in individuals with more general measures of functional impairment and may also relate to the predominance of lower (vs upper) extremity joint disease. Functional status specific to individual joints and to upper and lower extremity activities was measured on HJHS and HAL, respectively, and demonstrated expectedly strong correlations between HJHS Ankle scores and HAL Lying/Sitting/Kneeling/Standing, Functions of the Legs, and Complex Lower Extremity Activities. Although HJHS Elbow and Knee scores reached strong (but not the strongest) levels of correlation with arm- or leg-specific HAL domain scores, these results seem to reflect the greater levels of pain associated with ankles versus knees and elbows in this population of PWH, and Elbow and Knee scores may be expected to reach higher correlations in populations with more significant elbow and knee pain and joint disease. In addition to physical constructs known to be affected in PWH, high construct validity was observed for aspects of mental health measured on EQ-5D-5L and SF-36v2 instruments. High correlations were found between EQ-5D-5L Anxiety/Depression and SF-36v2 Social Functioning, Role Emotional, and Mental Health domains, suggesting that these global PRO instruments are valid tools for assessing mental health issues in adult PWH.

In addition to correlations between related domains (eg, pain vs pain, function vs function), high and very strong correlations were found between certain pain/function comparisons, consistent with a close relationship between pain and functional impairment. SF-36v2 Bodily Pain correlated highly with EQ-5D-5L Mobility and Usual Activities and with HAL Overall Activity, and EQ-5D-5L Pain/Discomfort correlated highly with HAL Overall Activity. More specific aspects of pain were investigated on BPI, and EQ-5D-5L Mobility was found to be correlated highly with the more general BPI domains (Average Pain and Current Pain), but not the extremes of Worst Pain or Least Pain; similarly, HAL Overall Activity correlated highly with Average Pain, Current Pain, and Worst Pain, but not with Least Pain. Analysis of joint-specific HJHS and HAL scores seem to indicate a greater contribution of lower extremity (particularly ankle) versus upper extremity limitations to overall physical 
function (EQ-5D-5L Usual Activities correlated highly with HAL Functions of the Legs and Basic Lower Extremity Activities, but not with Functions of the Arms or Upper Extremity Activities; HAL Overall Activity correlated highly with HJHS Ankle, but not with Elbow or Knee domain scores); however, these data may be influenced by the greater degree of pain (and presumably functional damage) observed in this population in ankles versus other joints. Interestingly, social functioning appears to be the only construct associated more highly with upper extremity versus lower extremity function (SF-36v2 Social Functioning correlated highly with HAL Functions of the Arms and Upper Extremity Activities, but not with Functions of the Legs, Basic Lower Extremity Activities, or Complex Lower Extremity Activities). Mental health outcomes do not seem to align highly with pain, functional impairment, or other general measures of HRQoL, as SF-36v2 Mental Health, Mental Health Summary, and Role Emotional scores correlated highly only with EQ-5D-5L Anxiety/Depression. The EQ-5D VAS, a general measure of health status "today," seems to be driven primarily by pain, as this score correlates highly with various assessments of pain (BPI Pain Severity, Pain Interference, and Current Pain, and SF-36v2 Bodily Pain), as well as SF-36v2 General Health and Physical Summary.

The high level of construct validity demonstrated through this analysis complements previously reported information regarding test-retest reliability of the $5 \mathrm{PRO}$ instruments in the P-FiQ study population. ${ }^{18}$ Of the 381 participants enrolled in P-FiQ, the first 187 were asked to participate in an optional retest of each PRO instrument at the completion of the comprehensive care visit ( $\sim 3-4$ hours after the initial PRO test), to compare responses given while in a similar non-bleeding state. Median concordance between responses was generally high (ranging from 54.5\% [BPI] to 100\% [IPAQ]), and all EQ-5D-5L, BPI, SF-36v2, and HAL domains reached the intraclass correlation coefficient threshold of 0.70 , indicating high reliability. Together, reliability and construct validity data suggest that these PRO instruments provide a valid measure of pain, functional impairment, and HRQoL outcomes and that the choice of instrument in the research or clinical care setting should be driven by factors other than reliability and validity, such as individual symptoms, treatment planning goals, outcome tracking objectives, and administrative burden. For example, instruments that are broad in scope such as EQ-5D-5L and SF-36v2 may be valuable as screening tools for pain, physical impairment, and mental health issues, and more specific tools such as BPI and HAL may be useful in the context of patients with known pain or functional problems, to inform treatment or physical therapy interventions aimed at improving HRQoL.

An important limitation of this study is a potentially limited relevance of the study population to that of the entire US PWH population. Because the study enrolled only adult male PWH with a history of joint pain or bleeding, PRO instruments cannot be assumed to accurately assess pain, functional impairment, and HRQoL constructs in women with hemophilia or others with less significant joint damage. The study population is also remarkable for a high number of participants being overweight or obese, which may be expected to affect outcomes related to physical activity and joint range of motion; however, whether this rate is comparable to that of the larger PWH population with joint pain or bleeding is unclear. Another important characteristic of the study population is the high level of pain in the ankles versus other joints, which, although consistent with expected sites of pain among $\mathrm{PWH},{ }^{25-27}$ may have resulted in a reduced sensitivity toward identifying correlations of domains relevant to non-ankle joint pain. An additional limitation of the study is the participation of subjects in the optional HJHS evaluation, which may not have been performed in some sites or circumstances due to considerations related to availability of a physical therapist trained in HJHS administration for the $\sim 1$-hour evaluation. However, the majority of correlations between HJHS domains and PRO scores reached statistical significance, and expected correlations between HJHS Ankle scores and relevant HAL domains reached a high correlation threshold of 0.5 , suggesting sufficient construct validity.

\section{Conclusion}

These data demonstrate high convergent validity and overall very strong correlations of related domains measuring pain, functional impairment, and anxiety/depression across PRO instruments in adult PWH. Each PRO instrument provides a different level of detail in describing HRQoL among PWH; the optimal choice of instrument to administer in the clinical or research setting may depend on the individual's symptoms, treatment planning goals, or outcome tracking research objectives, with the consideration of administrative burden.

\section{Acknowledgments}

The P-FiQ study was managed by Quintiles Real World and Late Phase, Boston, Massachusetts, and Rockville, Maryland, with statistical analyses provided by Jennifer James, Senior Biostatistician, and was supported financially by Novo Nordisk Inc. Writing assistance was provided by Anna Abt, PhD, of ETHOS Health Communications, Yardley, 
Pennsylvania, which was supported financially by Novo Nordisk Inc., Plainsboro, New Jersey, in compliance with international Good Publication Practice guidelines.

The abstract of this paper was presented at the ISPOR 21st Annual International Meeting as a poster presentation with interim findings. The poster's abstract was published in Value in Health (https://www.ispor.org/Scientific PresentationsDatabase/Presentation/63996).

\section{Disclosure}

KB has received grant/research support from Novo Nordisk Inc. and is a scientific advisor for Precision Health Economics. MR has received grant/research support from Baxter, Biogen Idec, Novo Nordisk Inc., and Pfizer and has served as a consultant to Kedrion and Novo Nordisk Inc. DLC is an employee of Novo Nordisk Inc. NNI is an employee of Novo Nordisk Inc. CLK has served as a consultant to Baxalta, Genentech, and Novo Nordisk Inc. and has received grant/ research support from Novo Nordisk Inc. The authors report no other conflicts of interest in this work.

\section{References}

1. Franchini M, Mannucci PM. Past, present and future of hemophilia: a narrative review. Orphanet J Rare Dis. 2012;7:24.

2. Konkle BA, Josephson NC, Nakaya Fletcher S. Hemophilia B. In: Pagon RA, Adam MP, Ardinger HH, et al., editors. GeneReviews (R). Seattle, WA: University of Washington; 1993.

3. Konkle BA, Josephson NC, Nakaya Fletcher S. Hemophilia A. In: Pagon RA, Adam MP, Ardinger HH, et al., editors. GeneReviews $(R)$. Seattle, WA: University of Washington; 1993.

4. Roosendaal G, Lafeber FP. Blood-induced joint damage in hemophilia. Semin Thromb Hemost. 2003;29(1):37-42.

5. Roosendaal G, Lafeber FP. Pathogenesis of haemophilic arthropathy. Haemophilia. 2006;12(Suppl 3):117-121.

6. Riley RR, Witkop M, Hellman E, Akins S. Assessment and management of pain in haemophilia patients. Haemophilia. 2011;17(6):839-845.

7. Bullinger M, Globe D, Wasserman J, Young NL, von Mackensen S. Challenges of patient-reported outcome assessment in hemophilia care-a state of the art review. Value Health. 2009;12(5):808-820.

8. de Moerloose P, Fischer K, Lambert T, et al. Recommendations for assessment, monitoring and follow-up of patients with haemophilia. Haemophilia. 2012;18(3):319-325.

9. Srivastava A. Haemophilia care - beyond the treatment guidelines. Haemophilia. 2014;20(Suppl 4):4-10.

10. Humphries TJ, Kessler CM. The challenge of pain evaluation in haemophilia: can pain evaluation and quantification be improved by using pain instruments from other clinical situations? Haemophilia. 2013;19(2):181-187.
11. Breivik H, Borchgrevink PC, Allen SM, et al. Assessment of pain. Br J Anaesth. 2008;101(1):17-24.

12. Cleeland CS. The Brief Pain Inventory: User Guide. Houston, TX: MD Anderson Cancer Center; 2009.

13. Keller S, Bann CM, Dodd SL, Schein J, Mendoza TR, Cleeland CS. Validity of the brief pain inventory for use in documenting the outcomes of patients with noncancer pain. Clin J Pain. 2004;20(5):309-318.

14. Krebs EE, Bair MJ, Damush TM, Tu W, Wu J, Kroenke K. Comparative responsiveness of pain outcome measures among primary care patients with musculoskeletal pain. Med Care. 2010;48(11):1007-1014.

15. van Genderen FR, van Meeteren NL, van der Bom JG, et al. Functional consequences of haemophilia in adults: the development of the Haemophilia Activities List. Haemophilia. 2004;10(5):565-571.

16. van Genderen FR, Westers P, Heijnen L, et al. Measuring patients' perceptions on their functional abilities: validation of the Haemophilia Activities List. Haemophilia. 2006;12(1):36-46.

17. Kempton CL, Recht M, Neff A, et al. Impact of pain and functional impairment in US adult people with hemophilia (PWH): patient-reported outcomes and musculoskeletal evaluation in the pain, functional impairment, and quality of life (P-FiQ) study. Blood. 2015;126(23):39.

18. Wang M, Neff A, Shapiro A, et al. Reliability of patient-reported outcome (PRO) instruments in US adult people with hemophilia (PWH): the pain, functional impairment, and quality of life (P-FIQ) study. J Thromb Haemost. 2015;13:363.

19. EuroQol Group. EQ-5D-5L User Guide: Basic Information on How to Use the EQ-5D-5L Instrument. Version 2.1. The Netherlands: EuroQol Group; 2015. Available from: http://www.euroqol.org/ fileadmin/user_upload/Documenten/PDF/Folders_Flyers/EQ-5D-5L_ UserGuide_2015.pdf. Accessed March 28, 2017.

20. Maruish ME. User's Manual for the SF-36v2 Health Survey. 3rd ed. Lincoln, RI: Quality Metric Inc; 2011.

21. Feldman BM, Funk S, Lundin B, Doria AS, Ljung R, Blanchette V. Musculoskeletal measurement tools from the International Prophylaxis Study Group (IPSG). Haemophilia. 2008;14(Suppl 3):162-169.

22. Feldman BM, Funk SM, Bergstrom BM, et al. Validation of a new pediatric joint scoring system from the International Hemophilia Prophylaxis Study Group: validity of the hemophilia joint health score. Arthritis Care Res. 2011;63(2):223-230.

23. Cohen J. A power primer. Psychol Bull. 1992;112(1):155-159.

24. Sherlock E, O'Donnell JS, White B, Blake C. Physical activity levels and participation in sport in Irish people with haemophilia. Haemophilia. 2010;16(1):e202-e209.

25. Gamble JG, Bellah J, Rinsky LA, Glader B. Arthropathy of the ankle in hemophilia. J Bone Joint Surg Am. 1991;73(7):1008-1015.

26. Hermans C, Dolan G, Jennings I, et al. Managing haemophilia for life: 5th Haemophilia Global Summit. Eur J Haematol. 2015; 95(Suppl 78):1-25.

27. Stephensen D, Tait RC, Brodie N, et al. Changing patterns of bleeding in patients with severe haemophilia A. Haemophilia. 2009;15(6): $1210-1214$. 


\section{Supplementary materials}

List of independent ethics committees/ institutional review boards

1. Munson Medical Center 1105 Sixth Street Traverse City, MI 49684-2386, USA

2. Emory University 1599 Clifton Road, 5th Floor Atlanta, GA 30322, USA

3. St Vincent Hospital and Health Care Center, Inc. 8402 Harcourt Road, Suite 805 Indianapolis, IN 46260, USA

4. Western Institutional Review Board 3535 7th Avenue SW Olympia, WA 98502-5010, USA

5. Henry Ford Health System CFP-Basement 0462799 West Grand Boulevard Detroit, Ml 48202-2689, USA Chairperson: Dr Timothy Roehrs, PhD

6. Vanderbilt University 504 Oxford House Nashville, TN 37232-4315, USA

7. Western Institutional Review Board 1019 39th Avenue SE Puyallup, WA 98374, USA

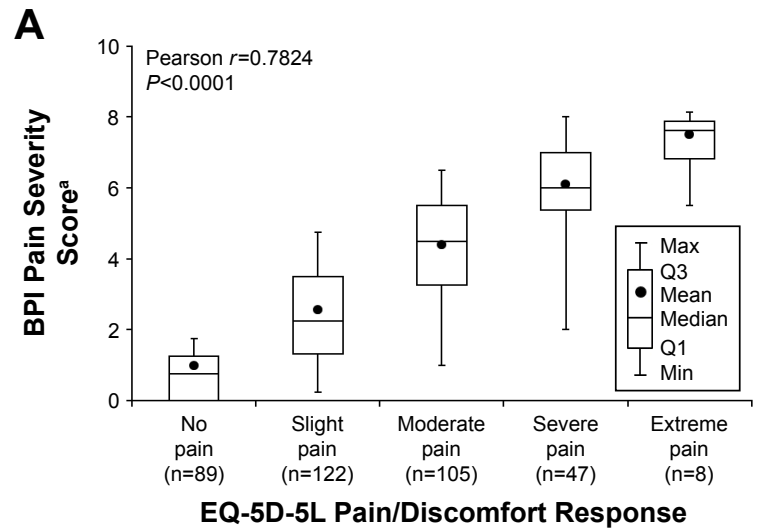

8. University of Colorado Multiple Institutional Review Board 13001 E. 17th Place, Building 500, Room N3214 Aurora, CO 80045, USA

9. Michigan State University Olds Hall 408 West Circle Drive, \#207 East Lansing, MI 48824, USA Chairperson: Dr Ashir Kumar

10. Oregon Health \& Science University 3181 SW Sam Jackson Park Road Portland, OR 97239-3098, USA

11. University of Minnesota D528 Mayo Memorial Building 420 Delaware Street S.E., MMC 820 Minneapolis, MN 55455, USA

12. Rush University Medical Center 1653 West Congress Parkway Chicago, IL 60612-3833, USA

13. Wake Forest University Health Services Medical Center Boulevard Winston-Salem, NC 27157-1023, USA

14. Georgetown University 37 th and O Streets, NW Washington, DC 20057, USA.

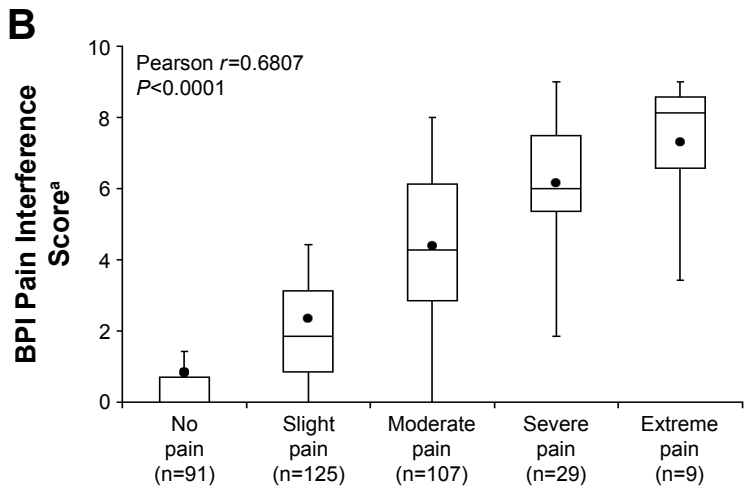

EQ-5D-5L Pain/Discomfort Response

Figure SI Correlation of EQ-5D-5L Pain/Discomfort with BPI Pain Severity (A) and Pain Interference (B).

Notes: $\mathrm{BPI}$ pain scores measured on a scale from 0 to 10; higher scores indicate greater pain severity or pain interference.

Abbreviation: BPI, Brief Pain Inventory v2 Short Form. 


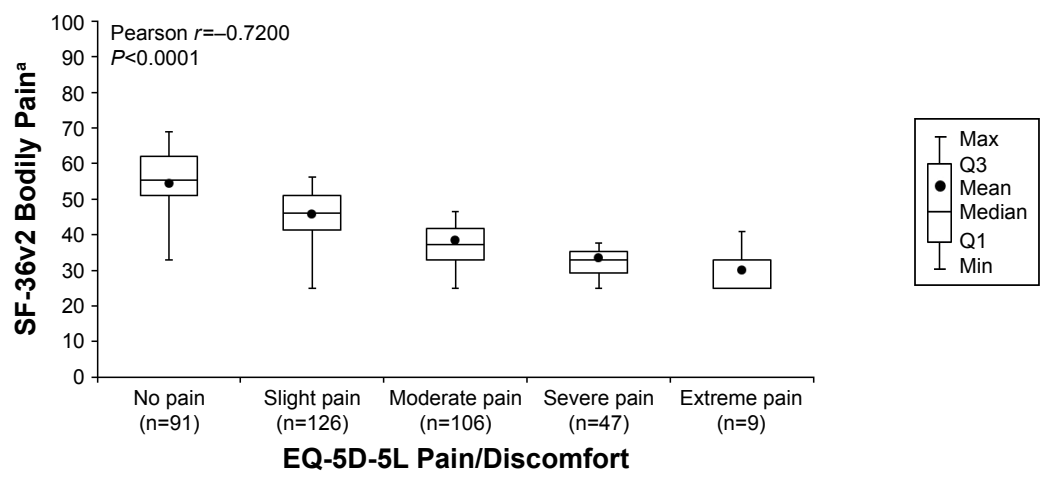

Figure S2 Correlation of EQ-5D-5L Pain/Discomfort with SF-36v2 Bodily Pain.

Notes: ${ }^{a}$ SF-36v2 Bodily Pain score measured on a scale from 0 to 100; lower scores indicate greater bodily pain.

A

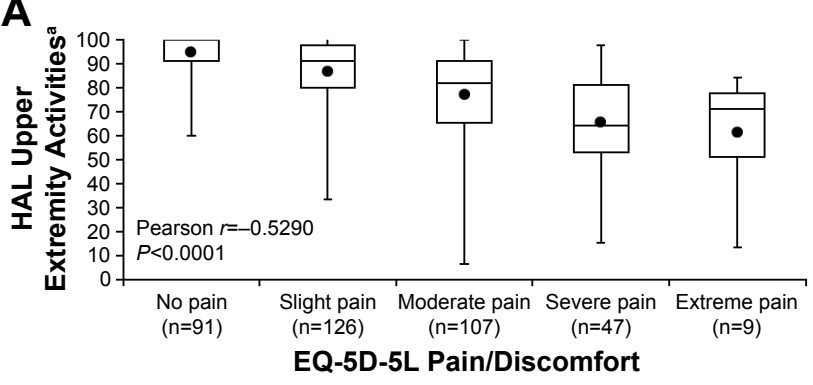

C

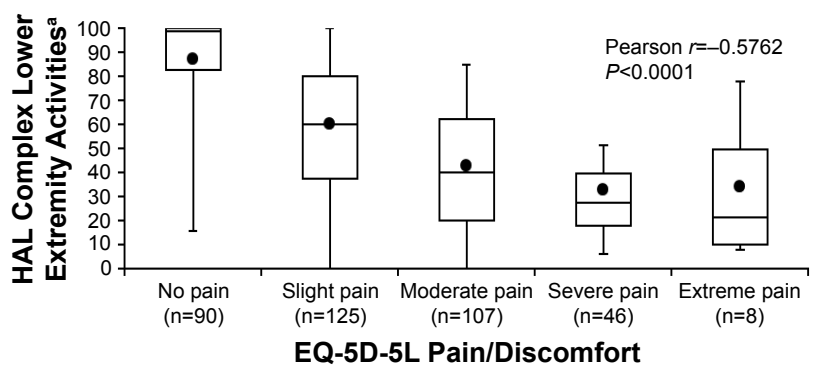

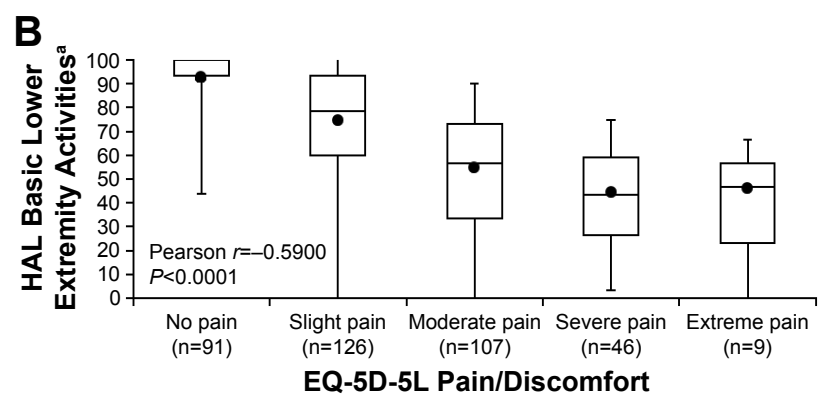

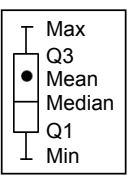

Figure S3 Correlation of EQ-5D-5L Pain/Discomfort with HAL Upper Extremity Activities (A), Basic Lower Extremity Activities (B), and Complex Lower Extremity Activities (C).

Notes: ${ }^{a} \mathrm{HAL}$ scores measured on a scale from 0 to 100; higher scores indicate better functional status.

Abbreviation: HAL, Hemophilia Activities List.

Patient Preference and Adherence

\section{Dovepress}

\section{Publish your work in this journal}

Patient Preference and Adherence is an international, peer-reviewed, open access journal that focuses on the growing importance of patient preference and adherence throughout the therapeutic continuum. Patient satisfaction, acceptability, quality of life, compliance, persistence and their role in developing new therapeutic modalities and compounds to optimize

clinical outcomes for existing disease states are major areas of interest for the journal. This journal has been accepted for indexing on PubMed Central. The manuscript management system is completely online and includes a very quick and fair peer-review system, which is all easy to use. Visit http://www. dovepress.com/testimonials.php to read real quotes from published authors. 